\title{
A PROBLEM OF MARTIN CONCERNING STRONGLY CONVEX METRICS ON $E^{3}$
}

\author{
E. D. TYMCHATYN ${ }^{1}$ AND B. O. FRIBERG
}

\begin{abstract}
If $d$ is a strongly convex.metric on $E^{3}$ and $C$ is a simple closed curve in $E^{3}$ such that $C$ is the union of three line segments then $C$ is unknotted.
\end{abstract}

In a talk presented to a Topology Conference at Arizona State University in 1967, Joseph Martin asked the following question: If $d$ is a strongly convex metric on Euclidean 3-space $E^{3}$ and $C$ is a simple closed curve in $E^{3}$ that is the union of three line segments (with respect to the metric $d$ ) is $C$ unknotted? The purpose of this note is to answer his question in the affirmative.

A partially ordered space is a space $X$ together with a partial order $\leqq$ on $X$ such that $\leqq$ has a closed graph.

For $x \in X$ we let

$$
L(x)=\{y \in X \mid y \leqq x\} \text { and } M(x)=\{y \in x \mid x \leqq y\} .
$$

A chain is a totally ordered set. An order arc is a compact and connected chain. An antichain is a set which contains no nondegenerate chain. A set $A$ is a maximal antichain of $X$ if $A$ is an antichain and for each $x \in X$, there exists $y \in A$ such that either $x \leqq y$ or $y \leqq x$. An element $\theta \in X$ is called the zero (resp. identity) of $X$ if $\theta \leqq x$ (resp. $x \leqq \theta$ ) for each $x \in X$.

We shall need the following result which is a slight generalization of Theorem 2.6 in [5].

THEOREM 1. Let $X$ be a compact metric partially ordered space such that $X$ has a zero and an identity and, for each $x \in X, L(x) \cup M(x)$ is a connected set. If $A$ is a compact antichain in $X$ then $A$ is contained in a compact maximal antichain of $X$.

Proof. Let $A$ be a compact antichain in $X$ which is neither the zero nor the identity of $X$. Let $Y$ be the quotient space obtained from $X$ by

Presented to the Society, January 15, 1974; received by the editors July 20, 1973. AMS (MOS) subject classifications (1970). Primary 54F05, 55A25.

${ }^{1}$ The first named author was supported in part by National Research Council Grant A5616.

(c) American Mathematical Society 1974 
identifying the set $A$ to a point and let $\pi$ be the natural projection of $X$ onto $Y$.

Let $\leqq$ be the smallest partial order on $Y$ that makes $\pi$ an order preserving function, i.e. for $x \in X$ let

$$
\begin{aligned}
L(\pi(x)) & =\pi(L(x)) \text { if } A \cap L(x)=\varnothing \\
& =\bigcup\{\pi(L(y)) \mid y \in A \cup\{x\}\} \text { otherwise. }
\end{aligned}
$$

Then $\leqq$ has a closed graph, $Y$ has a zero and an identity, and, for each $y \in Y, L(y) \cup M(y)$ is connected. By [5, Theorem 2.6] there exists a compact maximal antichain $C$ of $Y$ such that $\pi(A) \subset C$. Hence, $\pi^{-1}(C)$ is a compact maximal antichain of $X$ which contains $A$.

Lemma 2. Let $B$ denote the closed unit ball with center at the origin $\theta$ in $E^{3}$. Let $A$ be an arc in $B$ such that, for each $\varepsilon$ with $0<\varepsilon \leqq 1, A$ meets the 2-sphere $S_{\varepsilon}$ with center at the origin and radius $\varepsilon$ in exactly two points. Then there is a homeomorphism $h$ of $B$ onto $B$ such that $h$ carries each $S_{\varepsilon}$ onto itself and $h$ carries $A$ onto a diameter of $B$.

Proof. Let $A_{+}$and $A_{-}$be the two half arcs determined by $A$, i.e. $A=A_{+} \cup A_{-}, A_{+} \cap A_{-}=\theta$. Let $\phi:(0,1] \rightarrow S_{1}$ be the map determined by $A_{+}$, i.e., $\phi(\varepsilon)=\pi_{\varepsilon}\left(A_{+} \cap S_{\varepsilon}\right)$, where $\pi_{\varepsilon}: S_{\varepsilon} \rightarrow S_{1}$ is the natural radial projection. Since the orthogonal group $O(3)$ is a bundle over the 2-sphere $S_{1}$ (see $[4$, p. 33]), $\phi$ lifts to a map $\Phi:(0,1] \rightarrow O(3)$. Setting

$$
\left.h_{1}\right|_{S_{\varepsilon}}=\pi_{\varepsilon}^{-1} \circ\left(\left.\Phi(\varepsilon)\right|_{S_{1}}\right)^{-1} \circ \pi_{\varepsilon}, \quad h_{1}(\theta)=\theta,
$$

defines a homeomorphism $h_{1}$ of $B$ onto itself which takes $A_{+}$onto a radius. Let $R^{2}$ be the Euclidean plane. The complement of $h_{1}\left(A_{+}\right)$in $B$ is homeomorphic to $(0,1] \times R^{2}$ by a homeomorphism which carries $S_{\varepsilon}$ onto $\{\varepsilon\} \times R^{2}$ for each $\varepsilon$ in $(0,1]$. Let $h_{2}$ be the homeomorphism of $B$ onto itself (fixed on $h_{1}\left(A_{-}\right)$) which, by translating each $\{\varepsilon\} \times R^{2}$ onto itself according to $h_{1}\left(A_{-}\right)$, takes $h_{1}\left(A_{-}\right)$onto the opposite radius. Now $h=h_{2} \circ h_{1}$ satisfies the conclusion of the lemma.

THEOREM 3. Let $\leqq$ be a partial order with closed graph on $E^{3}$ such that $\theta$ is the zero of $E^{3}$ and, for each $x \in E^{3}, L(x)$ is an order arc. If $A$ is a simple closed curve in $E^{3}$ such that $A=A_{1} \cup A_{2} \cup A_{3}$ where $A_{1}$ and $A_{2}$ are order arcs, $A_{1} \cap A_{2}=\{\theta\}$ and $A_{3}$ is an antichain, then $A$ is tame and unknotted.

Proof. Let $p \notin E^{3}$ and let $S^{3}=E^{3} \cup\{p\}$ be the one-point compactification of $E^{3}$. Extend the partial order $\leqq$ on $E^{3}$ to a partial order (which we again denote by $\leqq$ ) with closed graph on $S^{3}$ by making $p$ the largest element of $S^{3}$. Then $S^{3}$ with this partial order satisfies the hypotheses of Theorem 1. 
By Theorem 1 there is a compact maximal antichain $C$ of $S^{3}$ such that $A_{3} \subset C$. By the proof of Theorem 3 in [6] every compact maximal antichain of $E^{3}-\{\theta\}$ is a tame 2-sphere and there is a homeomorphism $h$ of $L(C)=\bigcup\{L(x) \mid x \in C\}$ onto the unit ball $B$ with center at the origin in $E^{3}$ such that, for each $\varepsilon$ with $0<\varepsilon \leqq 1, h^{-1}\left(S_{\varepsilon}\right)$ is a compact maximal antichain of $E^{3}$ where $S_{\varepsilon}$ is the sphere with center $\theta$ and radius $\varepsilon$ in $B$. Thus, for each $i=1,2$ and for each $\varepsilon$ with $0<\varepsilon \leqq 1, h^{-1}\left(S_{\varepsilon}\right) \cap A_{i}$ consists of exactly one point.

By Lemma 2 there is a homeomorphism $j$ of $B$ onto itself such that, for each $\varepsilon$ with $0<\varepsilon \leqq 1, j\left(S_{\varepsilon}\right)=S_{\varepsilon}$ and $j\left(h\left(A_{1}\right) \cup h\left(A_{2}\right)\right)$ is a diameter of $B$. Now, $j(h(A))$ is the union of a diameter $j\left(h\left(A_{1} \cup A_{2}\right)\right)$ of $B$ together with an $\operatorname{arc} j\left(h\left(A_{3}\right)\right)$ on the boundary of $B$. Thus, $j(h(A))$ is tame and unknotted in $B$ and hence $A$ is tame and unknotted in $E^{3}$.

A metric $d$ for a topological space $X$ is said to be strongly convex if for each $x, y \in X$ there exists a unique $z \in X$ such that $d(x, y) / 2=d(x, z)=$ $d(y, z)$.

A line segment in a metric space $(X, d)$ is a set isometric to a segment of the real line with its usual metric.

A metric $d$ for a compact space $X$ is strongly convex if and only if each pair of points $x$ and $y$ of $X$ is contained in a unique minimal line segment $x y$ (see [2]).

THEOREM 4. Let $d$ be a strongly convex metric for $E^{3}$. If $C$ is a simple closed curve in $E^{3}$ such that $C$ is the union of three line segments then $C$ is tame and unknotted.

Proof. Suppose $C$ is composed of the three line segments $a b, a c$ and $b c$. Let $\leqq$ be the partial order on $E^{3}$ defined by setting $x \leqq y$ if and only if $x \in$ ay. Then (see [6]) $\leqq$ has a closed graph, zero $a$, and, for each $x \in E^{3}, L(x)$ is an order arc.

By the proof of Theorem 3 it follows that $a b \cup a c$ is a tame arc. Since $a$ was arbitrary, $C$ is locally tame. By [1] $C$ is tame.

Let $S=\left\{x \in E^{3} \mid d(x, a)=1\right\}$. By the proof of Theorem $3, S$ is a 2-sphere and $B=L(S)$ is a 3-cell. Rolfsen proved in [3] that, for each $x \in B-\{a\}, M(x) \cap S$ is a proper subcontinuum of $S$ which does not separate $S$. We may suppose without loss of generality that $C \subset B-S$.

If $x, y \in b c$ such that $x<y$ then $x y \subset b c$. Let $X$ be the quotient space obtained from $B$ by identifying each line segment $x y \subset b c$ such that $x<y$ to a point. Let $\pi$ be the natural projection of $B$ onto $X$. Then $X$ is a compact metric space. The partial order on $B$ induces in a natural way a partial order $\leqq$ ' on $X$ such that $\leqq$ ' has closed graph, zero $a$, set of maximal elements $S$; for each $x \in X-\{a\}, L(x)$ is a nondegenerate order arc, and, for each $x \in X-\{a\},\{y \in S \mid x \leqq y\}$ is a proper subcontinuum of $S$ 
which does not separate $S$. By Theorem 5 in [6], $X$ is a 3-cell. Now, $\pi(C)$ is a simple closed curve in $X$ which satisfies the hypotheses of Theorem 3. Hence, $\pi(C)$ is tame and unknotted in $X$. Thus, the fundamental group of $X-\pi(C)$ is infinite cyclic. Since $\pi$ is a homeomorphism off of $C, B-C$ is homeomorphic to $X-\pi(C)$. In particular, the fundamental group of $B-C$ is infinite cyclic. Thus $C$ is unknotted in $B$ and hence in $E^{3}$.

\section{REFERENCES}

1. R. H. Bing, Locally tame sets are tame, Ann. of Math. (2) 59 (1954), 145-158. MR 15, 816.

2. Joseph Martin, Recent developments in the geometry of continuous curves, Topology Conference Arizona State University 1967, edited by E. E. Grace, Tempe, Arizona.

3. D. Rolfsen, Strongly convex metrics in cells, Bull. Amer. Math. Soc. 74 (1968), 171-175. MR 37 \#2180.

4. N. Steenrod, The topology of fibre bundles, Princeton Math. Series, vol. 14, Princeton Univ. Press, Princeton, N.J., 1951. MR 12, 522.

5. E. D. Tymchatyn, Antichains and products in partially ordered spaces, Trans. Amer. Math. Soc. 146 (1969), 511-520. MR 41 \#7642.

6. - Some order theoretic characterizations of the 3-cell, Colloq. Math. 24 (1972), 195-203.

Department of Mathematics, University of Saskatchewan, Saskatoon, CANADA 